\title{
Pentheus und Dionysos in den Bakchen: Die Grenzen des klaren Dialogs
}

\author{
Camille Semenzato
}

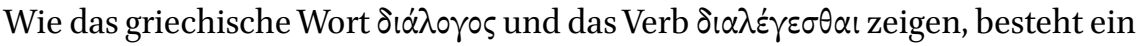
Dialog aus Worten ( $\lambda$ ó $\bigcirc \iota)$, die zwischen $(\delta\llcorner\alpha-)$ zwei oder mehreren Personen geäußert werden. ${ }^{1}$ Das Ziel eines solchen Gesprächs hängt von der Situation $\mathrm{ab}$, setzt aber ein gewisses offenes Verhalten der Beteiligten voraus, ohne dass sie sich am Ende unbedingt einigen und das Ziel auch tatsächlich erreichen müssen. Die sprachlichen Mittel, die sie verwenden, um sich zu verstehen und zu überzeugen, werden heutzutage von der Gesprächsanalyse untersucht. ${ }^{2}$ Der Dialog, den die zwei Hauptfiguren von Euripides' Bakchen miteinander führen, bietet ein sehr interessantes Beispiel, das aber von der Forschung selten behandelt wird: ${ }^{3}$ vielleicht weil dieser Dialog, auch wenn seine Struktur der gewöhnlichen Gesprächsorganisation entspricht, an seine Grenzen stößt, bis er tödlich scheitert? Ziel dieses Aufsatzes ist zu untersuchen, inwiefern der Sprachgebrauch selbst für dieses Scheitern verantwortlich ist.

\section{Ein Kommunikationsproblem}

Der Dialog der Bakchen ergibt sich aus einer problematischen Situation: Ein Fremder, ein Lydier, ist in Theben aufgetaucht. Er hat die thebanischen Frauen dazu verführt, ihre Männer, ihre Kinder, die Stadt sowie ihren Rang und Platz zu verlassen. Man erzählt, dass die Thebanerinnen (unter denen sich auch die Töchter des ehemaligen Königs, des berühmten Kadmos, finden) sich heimlich in den Bergen fragwürdigen sexuellen Handlungen hingeben unter dem Vorwand, dass sie einen neuen Gott, den sie Dionysos nennen, verehren. Die

1 Der Begriff wird hier in einem generellen Sinn gebraucht. Für die antike Gattung des Dialogs als Gespräch in Prosa siehe Jazdzewska (2014); Dubel (2015: 11-19).

2 Für die Anwendung der Gesprächsanalyse auf die griechische Tragödie siehe insbesondere Bonifazi u.a. (2016: III.4); Drummen (2017: 198-253); van Emde Boas (2017a, 2017b: 1-50).

3 Was die Gesprächsanalyse der Tragödie von Euripides betrifft, werden die Bakchen nach Schwinges Analyse (1968) selten erwähnt. Schuren (2015) zitiert zum Beispiel einige Stellen in Fußnoten, analysiert sie aber nicht gründlich. Drummen (2017:303-309) konzentriert sich auf die Verwendung von Partikeln. 
Ordnung und das Gleichgewicht des thebanischen Lebens werden durcheinandergebracht; Maßnahmen müssen ergriffen werden. Um die Situation zu klären und zu korrigieren, um das Problem zu lösen, initiiert der amtierende König Pentheus mit dem direkten Verantwortlichen dieser Unruhe, dem sogenannten Fremden - er wird einfach $\xi_{\varepsilon} \dot{v}_{0} \varsigma^{4}$ genannt -, den er hat verhaften lassen, einen Dialog.

Dieser Dialog wird das Problem aber nicht lösen, zumindest nicht in der Weise, wie Pentheus denkt. Auch wenn er im Laufe der Handlung vier Mal mit dem Fremden spricht, auch wenn beide während zweihundert Versen versuchen, sich zu verständigen und gegenseitig zu überzeugen, verstehen sie sich kaum. Es liegt, modern gesprochen, ein Kommunikationsproblem vor: Ein Problem mit schlimmen Folgen, da Pentheus schließlich einen furchtbaren Tod findet.

Heute würde ein externer Schlichter, der gerufen worden ist, um das Problem zu lösen, wahrscheinlich die unterschiedliche Herkunft der Beteiligten als erste mögliche Erklärung nennen: Pentheus ist Thebaner und spricht Griechisch; der Fremde kommt aus Lydien, spricht also Lydisch und ist ein sogenannter $\beta \alpha \dot{\rho} \beta \alpha \rho \rho_{0} .{ }^{5}$ In der Situation einer griechischen Tragödie gilt diese Erklärung aber nicht. Alle Figuren sprechen Griechisch, auch die fremdländischen. ${ }^{6}$ Dasselbe in den Bakchen: Die lydischen Frauen, aus denen der Chor besteht, ${ }^{7}$ erklären zwar einmal einem Boten, der sie nicht verstanden hat, dass sie als Fremde barbarische Lieder singen. ${ }^{8}$ Es ist aber mehr eine Ausrede: Der Chor hat sich gerade erlaubt, über Pentheus' Tod zu jubeln - ein Verhalten, das der Bote als Untertan des thebanischen Königs natürlich nicht verstehen kann. Ansonsten spricht der Chor griechisch. Fazit: Die Möglichkeit, dass der Fremde die griechische Sprache vielleicht nicht beherrscht, den üblichen Gebrauch der Sprache nicht kennt, und so Pentheus' Worte nicht versteht, ist keineswegs relevant.

4 Der griechische Text wird nach der Ausgabe von Diggle (1994) zitiert, außer wenn eine andere Lesart (der Handschriften L und P und des P.Ant. I 24) angegeben wird. Alle Übersetzungen sind meine.

5 Nach Hall (2004: 168) wird der Fremde durch seine Charakterisierung als छ́vvos durch Pentheus nicht als Barbar, sondern als "one of the eastern Greeks who were thought to have adopted the destructive luxury of the Lydians" betrachtet. Für die etymologische Analyse von $\beta \alpha \dot{\alpha} \beta \beta$ apos siehe Chantraine ( ${ }^{2} 1999$ : s.v.); Hall (2002: 112-117: Erörterung des onomatopoetischen Ursprungs).

6 Für die Ausländer bei Euripides siehe Saïd (1984).

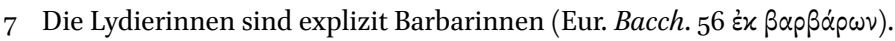

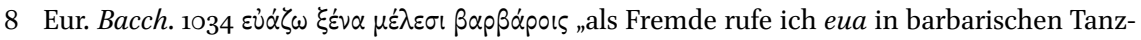
liedern". 
Eine zweite Erklärung könnte von der Natur des Fremden abhängen. Logischerweise denkt Pentheus, mit einem Menschen zu sprechen. Dank der generellen Hinweise, die im Prolog geäußert werden, wissen aber die Leser wie die Zuschauer, dass ein Gott hinter dem Fremden steckt. Und nicht irgendeiner: Dionysos selbst hat sich in den Fremden verwandelt. Die Kommunikationssituation und -schwierigkeiten werden selbstverständlich dadurch nicht vereinfacht. Dionysos präsentiert sich aber völlig menschlich ${ }^{9}$ und wird auch von Pentheus und den anderen Figuren für einen Menschen gehalten. Sogar die lydischen Frauen des Chors, welche als Anhängerinnen des dionysischen Kultes in Theben singen und tanzen, sehen im Fremden zwar ihren Anführer, aber nicht den Gott. Anders gesagt: Die doppelte Natur des Fremden als Mensch und Gott ist für die verschiedenen Figuren auf der reinen Handlungsebene nicht spürbar und so als Grund des Missverständnisses wenig relevant.

Deswegen wird für die folgende Analyse die Situation zuerst aus Pentheus' Sicht, dann aus demjenigen des Fremden betrachtet. Im Gegensatz zu Schwinge (1968: 339-433), der die Dialogszenen zwischen Pentheus und dem Fremden „Vom Standpunkt des alles überblickenden Zuschauers aus“ (342) ausführlich paraphrasiert, hat eine solche innovative Analyse den Vorteil, die Worte des einen und des anderen ohne Apriori oder Vorkenntnisse, die sonst in die Interpretation eingreifen, betrachten zu können, also die kommunikative Dynamik in den Blick zu nehmen. Auf diese Weise wird es vermieden, Pentheus von Anfang an als tyrannisch oder gar verrückt und somit an funktionierender Kommunikation nicht interessiert zu betrachten, oder aber die Worte des Fremden als typisch göttliche Äußerungen anzusehen, die dem Menschen Pentheus gar keine Verständnischance lassen.

Die leitenden Fragen sind die folgenden: Warum ist die Kommunikation zwischen Pentheus und seinem Gesprächspartner begrenzt? Wer ist dafür verantwortlich? Und aufgrund welchen (sprachlichen) Verhaltens? Die Analyse des Dialogs, der Konversationsdynamik, insbesondere der Redebeiträge und Sprecherwechsel ${ }^{10}$ im Zusammenhang mit dem Themenmanagement, sowie der Partikeln, welche auf diese Schritte und Wechsel hinweisen, wird herausstellen, inwiefern das Problem im Sprachgebrauch jedes Gesprächspartners, der mit seiner eigenen Weltanschauung zusammenhängt, liegt.

$9 \quad$ Eur. Bacch. 53-54.

10 Zu Redebeiträgen vgl. Meibauer (22001: 131-133). 


\section{Der Dialog als Lösung?}

Pentheus möchte die Situation, ihre Ursache, den Antrieb des Fremden verstehen. Deswegen lässt Pentheus ihn nach seiner Verhaftung nicht sofort töten, sondern stellt ihm zuerst eine Reihe von Fragen. Die Auseinandersetzung zwischen Pentheus und dem Fremden, die in Stichomythien und Distichomythien verfasst ist, ${ }^{11}$ besteht aus vier Teilen. Alle befinden sich in der Mitte der Tragödie, zwischen dem zweiten und dem vierten Epeisodion. Ihre Struktur und Rolle in der Handlung können folgendermaßen beschrieben werden:

\subsection{Erster Teil (Eur. Bacch. 460-5o8)}

Nach dem ersten Stasimon beginnt die zweite Szene mit den Worten eines Dieners, der am Ende der ersten Szene mit einigen Kollegen mit der Suche nach dem sogenannten Fremden beauftragt wurde. Nachdem der Diener unter anderem erklärt hat, wie der Fremde sich bei seiner Verhaftung benommen hat - er war erstaunlich ruhig und gutgelaunt -, beginnt Pentheus das Verhör seines Gefangenen.

Über den Fremden weiß Pentheus schon einiges. Er hat vom ihm und seinen Umtrieben gehört ${ }^{12}$ und sieht jetzt, inwiefern diese Gerüchte zutreffen: Anziehend, wie der Fremde aussieht, kann Pentheus gut verstehen, dass er die Frauen verführt. Diese äußerlichen Zeichen genügen dem König aber nicht: Das Versteckte interessiert ihn; er möchte all das Unklare klären. Deswegen fängt Pentheus, nachdem er das Aussehen des Fremden während sieben Versen kommentiert hat, ${ }^{13}$ damit an, Fragen zu stellen. Mit dem Eröffnungsmarker $\pi \rho \hat{\omega} \tau o v ~ \mu \varepsilon ́ v$ am Anfang des Verses 46o weist er klar darauf hin, dass er nun mit dem Gespräch beginnt; mit dem Imperativ $\mu \circ \iota \lambda \hat{\varepsilon} \xi \circ v$ gleich danach befiehlt er seinem Gesprächspartner, zu antworten. Auf diese erste Frage und ihre Antwort, welche die ersten Worte des Fremden darstellt, ${ }^{14}$ folgen siebenundvierzig Verse, welche die erste Dialogstelle bilden.

\subsection{Zweiter Teil (Eur. Bacch. 645-656)}

In der dritten Szene tritt Pentheus aufgeregt aus dem Palast: Der Fremde befindet sich nicht mehr im Gefängnis, wohin er nach der ersten Dialogstelle ge-

\footnotetext{
11 Die Standarddefinition der Stichomythie wurde von Gross (1905:9) formuliert. Siehe auch Seidensticker (1971: 183-184); Collard (1980); Schuren (2015: 1-4).

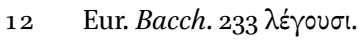

13 Eur. Bacch. 453-459.

14 Seine ersten Worte als Fremder, da der Gott Dionysos schon den ganzen Prolog gesprochen hat.
} 
bracht wurde. Da dieser ruhig vor dem Palast steht und auf den König warten scheint, nimmt Pentheus' Aufregung zu. Wie schon im ersten Gespräch versucht Pentheus, die unklare Situation mit deutlichen Fragen zu klären.

So beginnt dieser Abschnitt mit zwei von Pentheus nacheinander gestellten

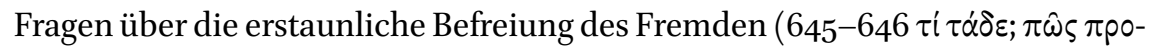
$\nu \omega^{\prime} \pi 10 \varsigma$ $\varphi \alpha^{\prime} i \eta \eta$; ,Was ist das? Wie erscheinst du am Eingang?"). Da sie so dicht und ohne bestimmtes Bindewort hintereinandergestellt sind, weisen sie auf Pentheus' Aufregung hin. Sie dienen zum Teil gleichzeitig der Aufforderung zum Sprecherwechsel: Die erste Frage stellt sich Pentheus selbst; die Verbform in der zweiten Person am Anfang des folgenden Verses zeigt deutlich, dass die zweite Frage an den Fremden gerichtet ist. Dieser wird auch gleich danach das Wort ergreifen. Nach elf Versen unterbricht aber das Eintreten eines Boten den Dialog. ${ }^{15}$

\subsection{Dritter Teil (Eur. Bacch. $787-846$ )}

Nach der Rede des Boten setzen Pentheus und der Fremde ihr Gespräch fort. Im Unterschied zu den vorigen Dialogstellen ist Pentheus' Wut nicht mehr auf den Fremden gerichtet. Ab diesem Moment ist er auf die in Wahnsinn versetzten Thebanerinnen konzentriert, nachdem der Bote ihre erstaunlichen und gewaltigen Taten beschrieben hat.

In dieser dritten Dialogstelle wird der Fremde aktiver als zuvor. Er übernimmt die Gesprächsleitung, indem er zum Beispiel anfängt zu sprechen, ohne

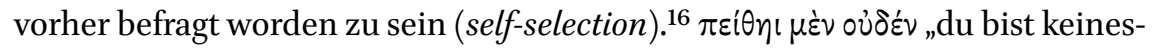
wegs überzeugt", sagt er am Anfang dieses Dialogteils (787) und weist, nachdem Pentheus den Boten angesprochen hat, ${ }^{17}$ mit $\mu \varepsilon^{\prime}$ darauf hin, dass er einen neuen Sprechakt plant und das Wort für eine Weile (fünf Verse) ergreift. ${ }^{18}$ Danach versucht er während 58 Versen, Pentheus zu überzeugen, nicht auf die Frauen zuzumarschieren. Seine Strategie basiert auf zwei Vorschlägen. Der erste - dass er die Frauen allein und unbewaffnet zurückbringt - ist angesichts von Pentheus' königlichem Status selbstverständlich ungeeignet. Der Fremde scheint ihn auch nur als Trick zu äußern, damit Pentheus den zweiten Vorschlag annimmt. Denn nachdem Pentheus den ersten Vorschlag mit einem

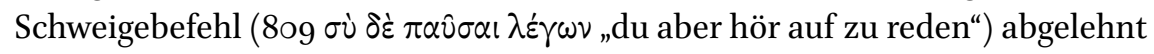

\footnotetext{
15 Die Länge dieser Stichomythie ist wahrscheinlich der Grund, warum sie nicht zu den von Schwinge (1968) bearbeiteten Stellen gehört. Als vierten Dialog analysiert er denjenigen zwischen Kadmos und Agaue, der für das Thema dieses Aufsatzes nicht relevant ist.

16 Vgl. zu diesem Phänomen Sacks u.a. (1974: 704).

17 Eur. Bacch. $77^{8-786 .}$

18 Bonifazi u.a. (2016: III.4 §28).
} 
hat - ein Befehl, der sich mit der ausdrücklichen Erwähnung von tig an den Fremden richtet, von diesem aber einfach ignoriert wird -, lockt der Fremde ihn mit der Äußerung seines innigsten Wunsches: die Frauen in den Bergen zu sehen. ${ }^{19}$ Diese Idee nimmt Pentheus nicht direkt an: Er muss sich vorher die Reise in allen Einzelheiten genau vorstellen können und stellt dafür wieder Fragen.

\subsection{Vierter Teil (Eur. Bacch. 918-972)}

Die vierte Dialogstelle erstreckt sich über 54 Verse in der vierten Szene: Der vom Fremden überzeugte Pentheus hat sich während des dritten Stasimons verkleidet. Als er neu gekleidet die Bühne wieder betritt, ist er bereit, dem Fremden zu folgen, um in die Berge zu den Frauen zu gehen.

Das Gespräch enthält keine Überredungsversuche oder -ziele mehr. Pentheus stellt zwar einige Fragen; sie dienen ihm aber alle dazu, zu versichern, dass seine Ausrüstung für eine Reise in die Berge angemessen ist, dass er gut und richtig aussieht. Von dieser Reise wird Pentheus aber nicht zurückkommen. Nachdem die erste Dialogstelle das erste Wort des Fremden dargestellt hat, bringt das Ende dieser vierten Stelle Pentheus' letzte Worte: ${ }^{20}$ fünf Verse in $\alpha \dot{\tau} \tau \lambda \alpha \beta \dot{\eta}$ welche das fehlende Verständnis zwischen den Gesprächspartnern augenfällig machen und so das Scheitern des Dialogs ankündigen. ${ }^{21}$

\section{3}

\section{Pentheus' Sprachgebrauch}

Wenn Pentheus spricht, tut er dies logischerweise nach seiner Art. Er stützt sich auf seine Kenntnisse, seine Einstellungen, kurz gesagt: auf seine Denkweise und Weltanschauung. Wichtig ist für ihn die Klarheit: was man deutlich sieht, in Kategorien einordnet, klar versteht, worauf man sich tatsächlich stützt. Im Dialog mit dem Fremden verwendet er wiederholt Vokabular der Klarheit und

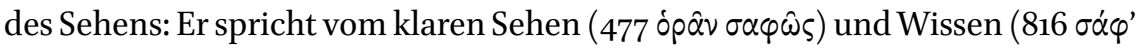

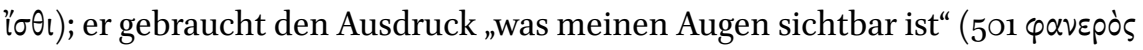

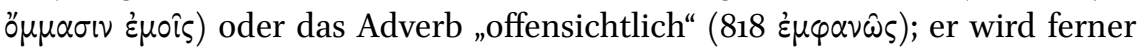

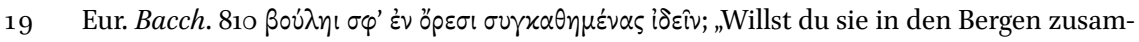
mensitzen sehen?" Der Fremde schlägt ganz genau Pentheus vor, heimlich und verkleidet in die Berge zu gehen, um die Frauen unbemerkt zu beobachten und so entscheiden zu können, ob sie wirklich schändlich handeln, bevor er mit ihnen schließlich nach Theben zurückkehrt.

20 Pentheus' letzte direkte Worte: Einige werden noch vom Boten (Eur. Bacch. 1059-1062, 1118-1121) und von Kadmos (1320-1322) wiedergegeben.

21 Siehe noch unter Abschnitt 6. 


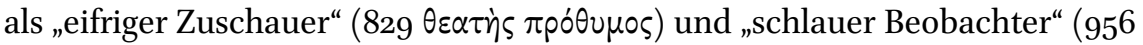

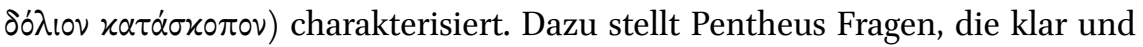
deutlich eingeleitet und gebildet werden. Sein Ziel ist, das Wer, Was, Wie und Warum zu bestimmen, um das für ihn Wichtige zu erfassen und einzuschätzen, zwischen den Möglichkeiten zu entscheiden und diese klassifizieren zu können.

In der ersten Dialogstelle gibt es zehn solche Fragen:22

[1] Euripides Bakchen 46o, 465, 467, 469, 471, 473, 477, 481, 485, 501

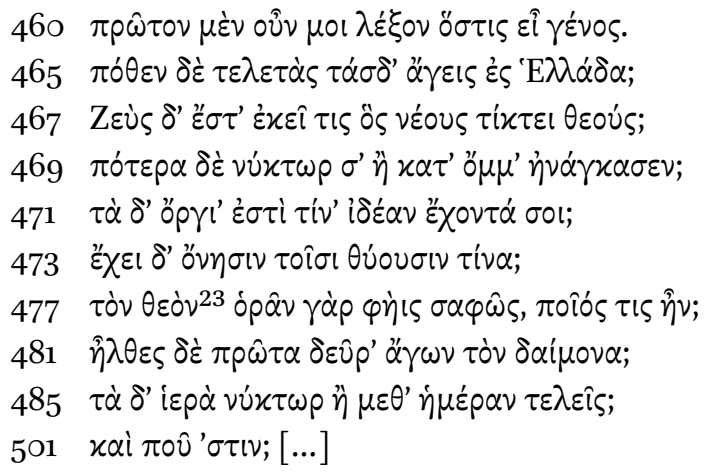

46o Sag mir also zuerst, welches Stamms du bist.

465 Woher bringst du diese Feiern nach Griechenland?

467 Ist dort ein Zeus, der neue Götter zeugt?

469 Hat er dich bei Nacht oder vor den Augen gezwungen?

471 Die Zeremonien, welches Äußere haben sie für dich?

473 Welchen Nutzen gibt es für die Opfernden?

477 Der Gott - du sagst nämlich, du siehst ihn klar-, was für einer war er?

481 Bist du zuerst hierher gekommen, die Gottheit bringend?

485 Die heiligen Dinge vollbringst du sie bei Tag oder bei Nacht?

501 Und wo ist er? [...]

Sieben dieser Fragen sind mit einem Interrogativpronomen oder -adverb gebil-

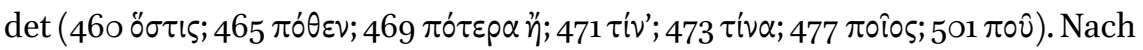
der ersten Frage, welche den Eröffnungsmarker $\pi \rho \hat{\omega} \tau o v ~ \mu \varepsilon^{\prime} v$ enthält, weisen

22 Pentheus stellt noch vier Fragen in der zweiten Dialogstelle sowie zwölf in der dritten und fünf in der vierten.

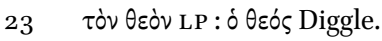


die fünf folgenden sowie noch zwei andere die Partikel $\delta \varepsilon ́$ auf, die einen Themawechsel markiert. ${ }^{24}$ Diese dauernden Wechsel deuten auch auf Pentheus' Führungsrolle hin: Sobald er eine Antwort bekommt, setzt er das Verhör mit einer anderen Frage über ein anderes Thema fort. ${ }^{25}$ In Vers 5 or hingegen markiert $x \alpha i$ die Fortsetzung des vorigen Themas. Dasselbe in Vers 477, der sich auf die eben ausgesprochenen Worte des Fremden bezieht: Pentheus strebt nach Genauigkeit, indem er das letzte Wort des vorigen Verses ( $476 \theta \varepsilon \circ \hat{v})$ wiederholt

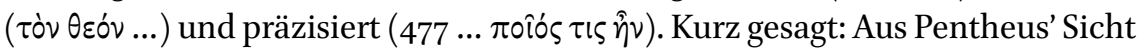
gibt es keinen Grund, dass ein so gut gegliederter Dialog die problematische Situation nicht klärt.

Pentheus' Sprachgebrauch zeigt, wer er ist: Der geschickte König, der die Vollmacht hat. Seinem Gefangenen gegenüber ist er der mächtigere (505 xupl$\omega \dot{\omega} \tau p \circ \varsigma \sigma \varepsilon \dot{\varepsilon} \theta v)$. Diese Selbstsicherheit ist in seinem Gebrauch der Verbformen sichtbar: Die Verben, die seine Taten ausdrücken, stehen im Futur: ${ }^{26}$ Als König weiß er genau, was er will. Er äußert auch zahlreiche Befehle im Imperativ, ${ }^{27}$ welche der Fremde oder seine Untertanen befolgen müssen. Im Allgemeinen zeigt er deutlich, dass er die Gesprächsthemen beherrscht. Obwohl er das spezifische Vokabular, das mit dem neuen Gott verbunden ist, nicht wirklich kennt, verwendet er es: Er hat von den $\tau \varepsilon \lambda \varepsilon \tau \alpha i$ gehört und gebraucht das Wort richtig. ${ }^{28}$ Wenn der Fremde von den öpүı $\alpha$ spricht, die er von seinem Gott bekommen hat (470), nutzt Pentheus sofort die Gelegenheit, um sich

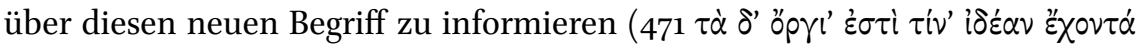
$\sigma 0 l ;) .{ }^{29}$

Die königliche Selbstsicherheit zeigt sich auch in Pentheus' Reaktion, nachdem der Fremde gesprochen hat: Seine Redebeiträge sind selten responsiv. ${ }^{30}$

\footnotetext{
24 Bonifazi u.a. (2016: III.4 §35).

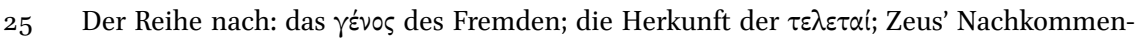
schaft; die Zeit der göttlichen Erscheinung; die Art und Form der őpүı $\alpha$; der Nutzen der Opfer; wie der Gott ist; die Reise des Fremden; der Ablauf der iєpó; wo der Gott steht.

26 Eur. Bacch. 493, 497, 792, 793, 796, 840, 843, 845, 846, 942, 954. Und außerhalb der Dialogstellen: 228, 232, 239, 240, 351, 512, 514 .

27 Eur. Bacch. 46o, 495, 503, 8o9, 816, 820, 934, 96o. Und außerhalb der Dialogstellen: 346, 35०, 352, 509, 511, 672, 780, 781 .

28 Eur. Bacch. 238 und 26o, 465 .

29 Diese Information, mit i $\delta \varepsilon \dot{\alpha} \alpha$ gebildet, gehört gewissermaßen auch zum Wortschatz des Sehens.

30 Bei Redebeiträgen (bzw., in seiner Terminologie, Gesprächsschritten) unterscheidet Mroczynski (2014: 76-78) zwischen iniitierenden (entsprechend in etwa dem englischen first pair-parts), respondierenden (second pair-parts) und reaktivierenden. Mit der früheren Literatur klassifiziert er die respondierenden in responsive, nonresponsive und teilresponsive Gesprächsschritte.
} 
Sobald eine Frage seiner Meinung nach geklärt ist, verweigert er die Fortsetzung des bisherigen Themas und führt ein neues ein.

Die Stelle, in der Dionysos' Herkunft diskutiert wird, ist ein gutes Beispiel:

[2] Euripides Bakchen 466-469

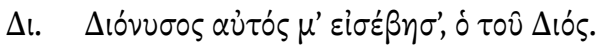

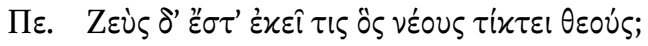

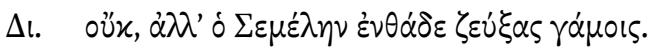

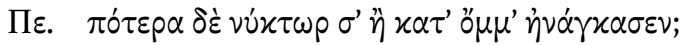

Di. Dionysos selbst hat mich eingeführt, der von Zeus.

Pe. Ist dort ein Zeus, der neue Götter zeugt?

Di. Nein, aber er hat sich hier mit Semele in Ehe vereint.

Pe. Hat er dich bei Nacht oder vor den Augen gezwungen?

Wenn der Fremde von Dionysos als Sohn des Zeus spricht, antwortet Pentheus ironisch mit einem reaktivierenden Gesprächsschritt, indem er gleichzeitig Zeus' Namen wiederholt (respondierte Komponente), mit $\delta \dot{\varepsilon}$ einen Themawechsel markiert und eine neue Frage stellt (initiierende Komponente). Wenn der Fremde aber bestätigt, dass der Dionysos, von dem er spricht, in Theben ( $\dot{\varepsilon} v \theta \alpha \dot{\delta} \delta \varepsilon)$ von Semele, Kadmos' Tochter und Pentheus' Tante, geboren worden ist, wechselt Pentheus ganz und gar das Thema: seine neue Frage - mit

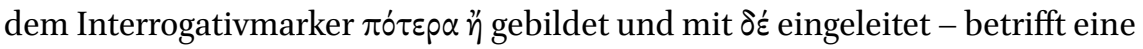
ganz neue Idee, die mit noch nicht verwendeten Wörtern geäußert wird. Denn die Frage nach Dionysos' Herkunft ist von da an für ihn geklärt. Da er schon vor seiner Begegnung mit dem Fremden gehört hat, dass dieser Dionysos für Zeus' göttlichen Sohn hält, ${ }^{31}$ und da der Seher Teiresias ihm auch die ganze Geschichte von Dionysos' Geburt erzählt hat, ${ }^{32}$ braucht er nichts mehr darüber zu wissen.

Pentheus' respondierte Gesprächsschritte sind oft nonresponsiv: ${ }^{33}$ Er ignoriert die Antworten des Fremden, als ob sie nicht geäußert worden wären, und weist damit die implizite Aufforderung zurück. Pentheus' dreiteilige Antwort auf die Frage des Fremden zu seiner Bestrafung könnte zum Beispiel sehr gut als ein ununterbrochener Redebeitrag geäußert werden:

31 Eur. Bacch. 242-243.

32 Eur. Bacch. 286-297.

33 Zur Terminologie s. o. Anm. 3 o. 
[3] Euripides Bakchen 493-499

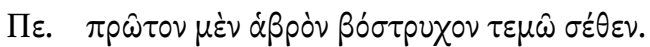

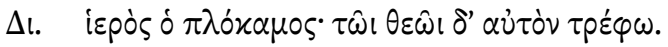

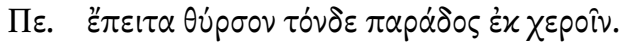

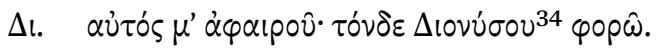

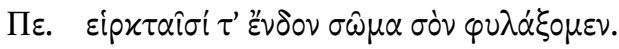

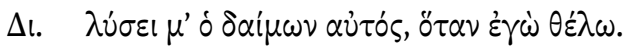

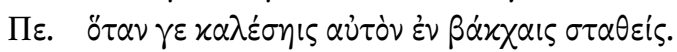

Pe. Zuerst werde ich deine zarten Locken schneiden.

Di. Mein Haar ist heilig; ich lasse es für den Gott wachsen.

Pe. Dann übergib mir diesen Thyrsos aus deinen Händen.

Di. Nimm ihn selbst weg; als den des Dionysos trage ich diesen.

Pe. Und wir werden deinen Körper im Gefängnis bewachen.

Di. Die Gottheit selbst wird mich befreien, wann ich es will.

Pe. Na ja, wann du ihn rufen wirst, in der Mitte der Bakchen stehend.

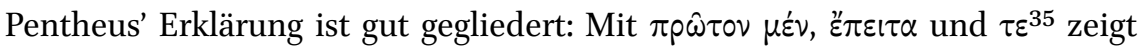
er klar, dass die Bestrafung, die er sich für den Fremden ausgedacht hat, drei Teile oder Momente hat. Pentheus' Beiträge sind alle vollständig, so dass jedes Mal ein unproblematischer Sprecherwechsel erfolgt. Sobald der Fremde fertig ist, setzt Pentheus aber seine Rede fort, ohne sich um seinen Gesprächspartner zu kümmern. Erst in Vers 499 zeigt er, dass er die Beiträge des Fremden oder zumindest den letzten - gehört hat: Er greift das zuletzt geäußerte Thema auf, indem er die Konjunktion ö $\tau \alpha \nu$ wiederholt, und wandelt es spöttisch - mit einem $\gamma \varepsilon$, das seinen Standpunkt markiert - um. ${ }^{36}$

Pentheus' Sprachgebrauch ist klar: Aus seiner Sicht gibt es kein anderes mögliches Verhalten, keinen einzigen Grund, sich und seine Sprache infrage zu stellen. Dank seinem Sprachgebrauch bekommt er die Antworten, die er erwartet hat, da sie Gerüchte über den Fremden bestätigen. Von diesem Sprachgebrauch ist Pentheus überzeugt, auch wenn er an die Grenzen stößt, welche

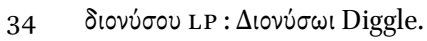

35 Nach Drummen (2017: 212 Anm. 37, 305 Anm. 113) wird ein solches initiierendes $\tau \varepsilon$ in der Tragödie selten geäußert.

36 Bonifazi u.a. (2016: III.4 §63). Nach Drummen (2017: 306), the addition of $\gamma \varepsilon$ in resonating utterances emphasizes the speaker's hostile goal in echoing his opponent's words, and thereby implies anger or hate. In diesem spezifischen Fall erfordert aber der Kontext, die Partikel als spöttisch zu interpretieren, wie die Kommentare zu den Bakchen (die Drummen in ihrer Anm. 114 auch zitiert) es betonen. Siehe auch Eur. Bacch. 796.
} 
der Fremde durch seine fehlende Angst vor der sicheren, klar ausgesprochenen Macht und durch seinen im Unterschied dazu mehrdeutigen Sprachgebrauch darstellt.

\section{Der Sprachgebrauch des Fremden}

Als Gefangener kooperiert der Fremde gut: Er wird nie wütend, er weigert sich nie, zu antworten. Ab und zu erlaubt er sich, den König zu korrigieren. ${ }^{37}$ Wenn diese Korrekturen einen nonresponsiven Gesprächsschritt darstellen, haben sie aber keine negativen Folgen. Sie helfen eher der Dialogfortsetzung, da Pentheus nie in Verlegenheit gebracht wird und immer weiß, wie er zu reagieren hat.

Im ersten Dialogteil ist der Fremde eher passiv: Er stellt mit dem Interrogativpronomen $\tau i$ eine einzige Frage, um zu erfahren, ${ }^{38}$ welche Bestrafung Pentheus für ihn bereithält. Niemals ergreift er die Initiative, das Thema zu wechseln. So enthalten seine ersten Gesprächsschritte keine initiierenden Marker. Dasselbe in der zweiten Dialogstelle und in der ersten Hälfte der dritten: Die zwei Fragen des Fremden sind beide mit den kurz vorher geäußerten Bemerkungen von Pentheus verbunden und fragen nach einer Präzisierung. ${ }^{39}$

Ab dem Vers 810 (in der zweiten Hälfte der dritten Dialogstelle) ${ }^{40}$ wird der Fremde aktiver: Er stellt mehr Fragen, verwendet mehr Partikeln, bringt dadurch seinen Standpunkt aktiver ins Gespräch ein. Diese markieren meistens keinen Themenwechsel, sondern helfen ihm, dem Gespräch die gewünschte Richtung zu geben. Denn als folgsamer Gefangener eines machtvollen Königs äußert er seine Ideen und Meinungen nie explizit. Als selbstbewusster Mensch, ${ }^{41}$ der vom Sprachgebrauch seines Gesprächspartners keineswegs beeindruckt ist, bringt er mit kleinen Hinweisen Pentheus aber dazu, seine Vorschläge und Meinungen anzunehmen. Zum Beispiel kurz nach diesem Wendepunkt:

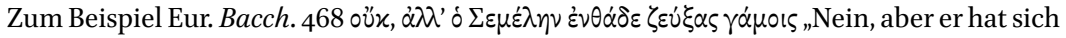
hier mit Semele ehelich vereinigt".

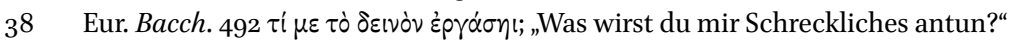

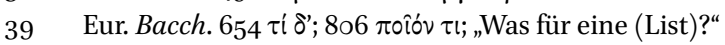

40 Der Ausruf $\hat{\alpha}$ in Vers 810 wird gewöhnlich (zum Beispiel Dodds 2¹96o: 175; Roux 1972: 493; Seaford 1996: 213; Susanetti 2010: 239) als Wendepunkt der Tragödie betrachtet, da der Fremde ab diesem Moment die Oberhand über Pentheus gewinnt.

41 Wie schon gesagt, spielt es hier keine Rolle, dass der Fremde eigentlich ein Gott ist. 
[4] Euripides Bakchen 816-818

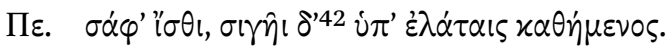

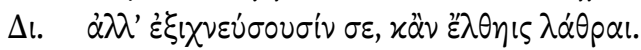

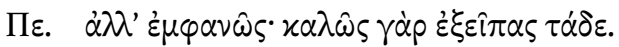

Pe. Wisse es klar, aber in Schweigen unter den Fichten sitzend.

Di. Sie werden dich aber aufspüren, auch wenn du unbemerkt kommst.

Pe. Dann sichtlich; denn du hast gut dazu gesprochen.

Mit $\sigma \alpha \varphi^{\prime}{ }^{\prime}{ }^{\prime} \sigma \theta_{l}$ bestätigt Pentheus, dass er die Frauen gerne sehen möchte, auch wenn diese vom Wein berauscht sind. Er fügt aber eine zusätzliche Information hinzu, die den vorigen mit einem adversativen $\delta \dot{\varepsilon}$ entgegengesetzt ist: $\mathrm{Er}$ möchte nicht gesehen werden. Da dies dem Plan des Fremden nicht entspricht, wählt dieser einen nonresponsiven Gesprächsschritt aus, den er mit $\dot{\alpha} \lambda \dot{\alpha}$ markiert und der die Unangemessenheit von Pentheus' Idee zeigt. So wird Pentheus dazu gebracht, seine erste Idee, wiederum mit $\dot{\alpha} \lambda \dot{\alpha}$, zu korrigieren: Er gibt die Absicht auf, zu schweigen, sich zu verstecken, unbemerkt zu bleiben; er will sich jetzt offen zeigen. Von dieser neuen Idee ist Pentheus ganz überzeugt; er dankt dem Fremden auch dafür: Sein Sprachgebrauch war richtig!

Im Vergleich zu Pentheus benützt der Fremde eine weniger klare Sprache. Er beantwortet zwar Pentheus' Fragen und beteiligt sich am Gespräch, scheint sich aber leicht daneben zu bewegen. Seine Antworten sind meistens indirekt, ungenau. Wenn Pentheus ihm im zweiten Dialogteil zum Beispiel fragt, wie

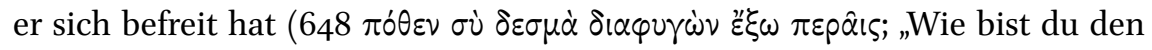
Fesseln entkommen und trittst heraus?"), weist der Fremde auf die anschei-

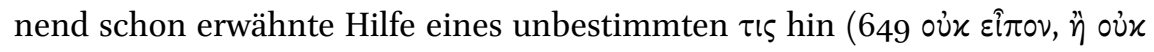

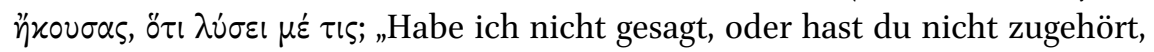
dass jemand mich befreien wird?"). Die zahlreichen Informationen, welche der Fremde als Sequenzerweiterungen (expansions) äußert, verkomplizieren auch die Struktur des Dialogs. Pentheus' allererste Frage betrifft logischerweise das

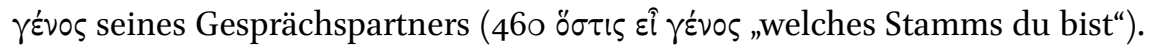
Die erwartete Antwort würde den Namen des Fremden mit demjenigen seines Vaters und/oder seiner Familie enthalten. Der Fremde wird aber nur von sei-

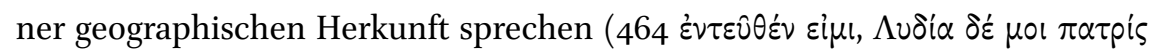
„Von dort bin ich, Lydien ist meine Heimat“), und dies erst vier Verse später. Dazwischen erweitert er die Gesprächssequenz mit einem reaktivierenden

$42 \quad \delta^{\prime} \mathrm{P}: \gamma^{\prime}$ Diggle. 
Gesprächsschritt, ${ }^{43}$ als ob sicherstellen möchte, dass Pentheus seine bevorstehende Antwort verstehen wird. Diese Erweiterung sowie die sofortige Antwort von Pentheus auf seine indirekt gestellte Frage, die mit dem Indefinitpronomen $\pi$ ov gebildet ist, ${ }^{44}$ erlauben ihm aber, nicht mehr als seine geographische Herkunft anzugeben und so ungenau zu bleiben. ${ }^{45}$

Dass der Fremde nur undeutlich antwortet und sogar ein Katz-und-Maus-Spiel treibt, merkt Pentheus schnell. Als selbstsicherer König lässt er sich nicht verunsichern. Aus seiner Sicht kann der Verantwortliche für die Unklarheiten in ihrem Dialog nur der Fremde sein. Und als mächtiger König lässt Pentheus es seinem Gesprächspartner auch wissen: Im ersten Dialogteil stellt er drei Mal mit einem expliziten Beweis fest, dass er den Versuch des Fremden, ihn irrezuführen, bemerkt hat:

1. In Vers 473 möchte Pentheus wissen, welchen Nutzen - eine klare Frage mit einem Interrogativpronomen, nach einem $\delta \dot{\varepsilon}$ als Themawechsel-

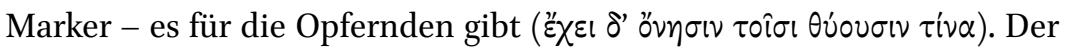
Fremde weigert sich zu antworten, indem er sich in einem nonresponsiven Gesprächsschritt hinter einem religiösen Grund versteckt: Die $\theta \varepsilon ́ \mu ı \zeta$,

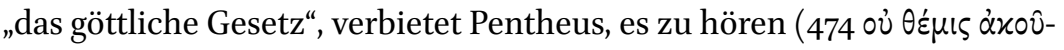
$\left.\sigma \alpha i \sigma^{\prime}\right)$. Dieser Grund entspricht einer sozialen Erwartung: Jeder weiß, dass es unziemlich ist, gegen die témuऽ zu handeln, dass man sonst eine ¿ßßpıৎ, eine „Freveltat" begehen würde. Der Fremde setzt das Versteckspiel aber fort, indem er noch hinzufügt, dass es eigentlich wertvoll ist, es zu

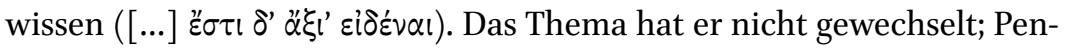
theus könnte noch darauf zurückgreifen. Als mächtiger König beschließt er aber, sich seinem Gefangenen nicht zu unterwerfen, sondern ihm zu zeigen, dass er seine Sprachspielerei bemerkt hat. Ohne sich zu ärgern,

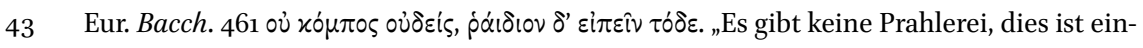
fach zu sagen." (oủ xó $\mu \pi \circ 5$ LP : oủx öxvos Diggle).

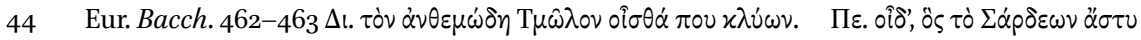

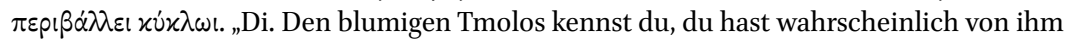
gehört. Pe. Ich weiß, derjenige, der sich im Kreis um die Stadt Sardes legt." Die lydische Herkunft des Fremden kennt Pentheus eigentlich schon. Sie gehört zu den Gerüchten, die

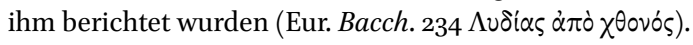

45 Für die überblickenden Zuschauer ist die Antwort auf diese Frage selbstverständlich ironisch, denn $\gamma \varepsilon$ v́vৎ spielt auch auf die doppelte Natur des Fremden als Gott und Mensch an. 
setzt er das Spiel fort. Er antwortet auf die Bedeutung von $\alpha \xi_{10 \varsigma}$ als das, was einen großen Wert oder einen angemessenen Preis hat, mit dem Verb $\varkappa \varkappa \delta \eta \lambda \varepsilon \varepsilon \dot{\varepsilon \varepsilon v}$, das „eine Münze durch Zusatz von minderwertigem Metall verfälschen "46 bedeutet: Der Fremde hat seine Argumente gut verfälscht,

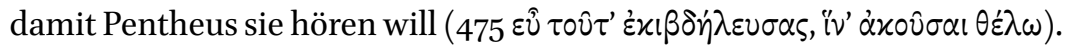
Der König wird sich aber nicht dazu herablassen.

2. In der Fortsetzung des Dialogs interessiert sich Pentheus nicht mehr für den Kult, sondern direkt für den Gott. Er möchte wissen, was für einer

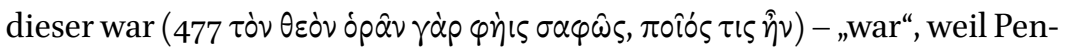
theus an den Moment denkt, in dem der Fremde seinem Gott begegnet ist, von ihm eingeweiht wurde, und der für Pentheus ganz klar zur Vergangenheit gehört. Als Antwort auf das Interrogativpronomen $\pi$ oîऽ wählt der Fremde das indefinite Interrogativpronomen ó $\pi \circ \hat{\imath} о \varsigma$ aus. So scheint seine Antwort responsiv zu sein, während sie nonresponsiv ist: Der Gott war, wer auch immer er wollte, ohne dass der Fremde etwas zu sagen hatte

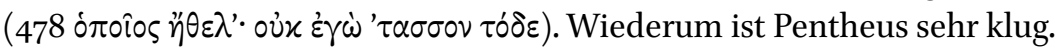
Er merkt selbstverständlich, dass sein Gesprächspartner seine Frage nur ungenau beantwortet hat, und lässt es wiederum mit einem klaren Verb

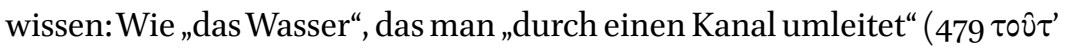
$\alpha \hat{\jmath} \pi \alpha \rho \omega \chi \varepsilon ́ \tau \varepsilon \cup \sigma \alpha \varsigma),{ }^{47}$ hat der Fremde Pentheus' Frage mit der Veränderung ins Indefinite abgelenkt.

3. Zehn Verse später äußert Pentheus wieder eine solche Bemerkung, mit der er das Verhalten und die Worte des Fremden beschreibt. Diese Beschreibung zerfällt in zwei Teile: Zuerst wirft Pentheus dem Fremden vor, schlechte $\sigma \circ \varphi i \sigma \mu \alpha \tau \alpha$ vorzubringen, wofür er bestraft werden muss (489

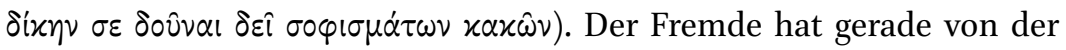

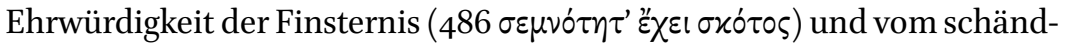

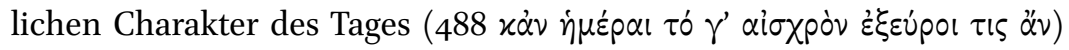
gesprochen. Zwei Charakterisierungen, die zu Pentheus' Begierde nach

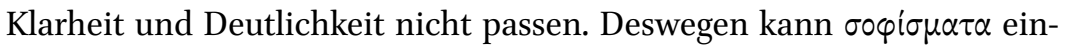
fach mit „Sophismen“ übersetzt werden: Pentheus wirft dem Fremden vor, schlechte Argumente zu äußern, die scheinbar logisch sind, die aber zu einem Fehlschluss führen. Und diejenigen des Fremden führen nicht nur zu einem Fehlschluss, sondern sind einfach $x \alpha x \alpha$, „schlecht“. Auch dafür muss der Fremde bestraft werden. ${ }^{48}$ Der Fremde reagiert sofort, indem

46 Siehe noch Eur. Med. 516, El. 550, Hipp. 616.

47 Siehe auch Eur. Supp. 1111, Plat. Leg. 844a.

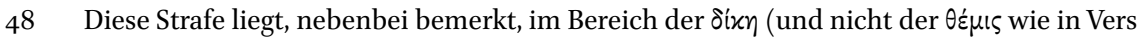

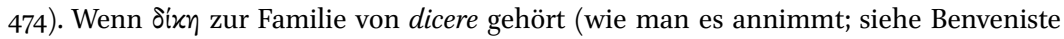


er auf die Satzstruktur von Pentheus zurückgreift, aber ein neues Objekt (mit $\delta \varepsilon \dot{)}$ ) hinzufügt: Auch Pentheus muss bestraft werden. Nicht wegen seiner guten oder schlechten Redekunst, sondern wegen seiner Unwissen-

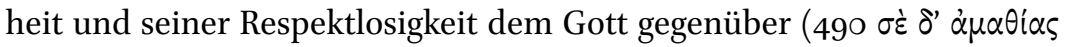

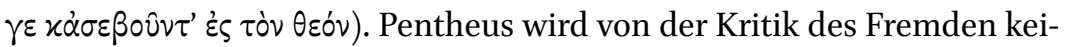
neswegs berührt. Er zeigt sich offen. Er anerkennt die Kühnheit ${ }^{49}$ und die

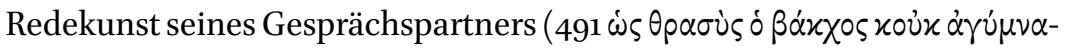
$\sigma \tau \circ \varsigma \lambda \gamma^{\prime}(\omega \nu)$. Und noch einmal zeigt er sich auch geschickt: Indem er die dritte Person braucht, verhindert er, dass der Fremde auf das scheinbare Kompliment direkt reagieren kann. ${ }^{50}$ Dieser muss (auf seine Art, ohne bestimmte Partikel) ein neues Thema einfügen und stellt so selbst eine Frage.

Aus der Sicht des Fremden ist aber Pentheus verantwortlich. Denn er strebt so sehr nach Klarheit, nach deutlichen Strukturen, dass er die mögliche Mehrdeutigkeit, die in den Worten steckt - und insbesondere in denjenigen des Fremden - nicht ernst nimmt. Dass ein Wort mehrere Bedeutungen besitzen kann, ist Pentheus klar. Am Ende der zweiten Dialogstelle zum Beispiel geht es um die Weisheit des Fremden:

\section{[5] Euripides Bakchen 655-656}

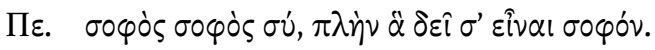

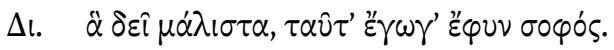

Pe. Weise, weise du, außer worin du weise sein musst.

Di. Worin es in ganz besonderem Maße sein muss, darin bin ich weise.

Pentheus nach ist der Fremde zwar weise, aber nicht in dem, worin er weise sein muss. Und dies betont er, indem er das Adjektiv zwei Mal wiederholt, am Anfang (noch mit dem Personalpronomen $\sigma u ́)^{51}$ und am Ende des Verses. Im Gegensatz dazu ist der Fremde überzeugt, genau darin weise zu sein. Und auch

1969: 107-110; Du Sablon 2014: 242), bedeutet das Wort eine „autoritative Aussage“, das „Recht“, die „Gerechtigkeit“, die ein König oder ein Richter verkörpert, den „Rechtsspruch“, den „Entschluss“ oder das „Urteil“, die man annehmen muss. Pentheus bemerkt aber nicht, dass seine $\delta \dot{x} x \eta$ weniger mächtig ist als die $\theta \dot{\varepsilon} \mu \iota \varsigma$, das „göttliche Gesetz“, das über die ganze Welt herrscht und sie reguliert.

49 Nach Roux (1972: 340) wird im letzten Drittel des fünften Jahrhunderts der Rhetor, der skrupellos eine falsche Rede halten kann, $\theta$ pa ús genannt.

50 Di Benedetto (2010: 370 ).

$5^{1} \quad$ Siehe auch Eur. Bacch. 186, Andr. 245. 
er zeigt es durch die Syntax seiner Antwort: Wie in einem Chiasmus greift er auf den Relativsatz zurück, ändert aber gleichzeitig $\sigma \varepsilon$ selbstverständlich zu है $\gamma \omega \gamma \varepsilon$

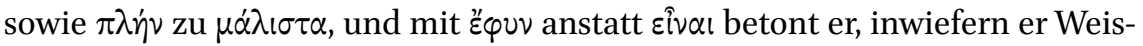
heit besitzt. ${ }^{52}$ Dass $\sigma \circ \varphi o ́ \varsigma$ zweideutig ist, wissen sowohl der Fremde als auch Pentheus: Die Wahl der Wortbedeutung hängt in diesem Fall von der Interpretation und Weltanschauung jeder Figur ab. ${ }^{53}$

Ein Wort kann aber auch gleichzeitig mehrere Bedeutungen ausdrücken, ohne dass es seine Kohärenz verliert. Ein gutes Beispiel dafür ist das Adjektiv $\delta \varepsilon เ v o ́ s$, das sowohl "furchtbar, schrecklich“ als auch "außerordentlich, erstaunlich" bedeutet. Als Einleitung zum zweiten Dialogteil, wenn Pentheus aus dem Palast kommt, spricht er von den $\delta \varepsilon เ \nu \alpha$, von denen er erfahren hat (642 $\pi \dot{\varepsilon} \pi \circ v \theta \alpha$

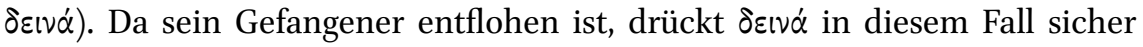
"schreckliche Dinge“ aus. Es sind gleichzeitig aber auch „außerordentliche, erstaunliche Dinge“, denn einen solchen Gefangenen hatte er noch nie. ${ }^{54}$

Deswegen ist Pentheus aus der Sicht des Fremden für das Scheitern ihres Dialogs verantwortlich: Er versteht die subtilen Unterschiede nicht, die den Sprachgebrauch des Fremden bilden und auf die Mehrdeutigkeit der Sprache hinweisen. Er hat den Eindruck, dass der Fremde immer neue Wörter beibringt

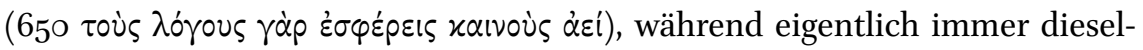
ben gebraucht werden. Damit der Dialog zur Lösung einer problematischen Situation wird, müssen die Gesprächspartner ein offenes Verhalten an den Tag legen. Pentheus kann aber keinen Kompromiss schließen. Er ist nicht bereit, den Sprachgebrauch und die Weltanschauung des anderen zu verstehen, nicht einmal zu akzeptieren. Zum Beispiel verwendet der Fremde, wenn er von seiner Einweihung spricht, mit der Verbform $\delta \delta \delta \omega \sigma \nu v$ ein Präsens, auch wenn die Handlung nicht zeitgleich zu seiner Äußerung stattfindet: Der Gott gibt dem

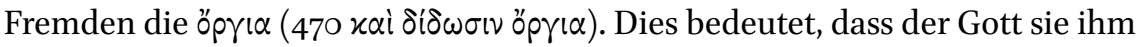
gegeben hat (so versteht es Pentheus, da er in Vers 477, wenn er gewissermaßen zu diesem Thema zurückkehrt, das Verb im Imperfekt gebraucht), aber auch, dass der Gott sie dem Fremden immer wieder gibt und immer wieder geben wird, so dass er sie ihm schlicht und einfach gibt. ${ }^{55}$ Die klaren Zeitstrukturen von Vergangenheit, Gegenwart und Zukunft, die Pentheus' Welt bilden, sind

\footnotetext{
52 Für diejenigen, die hinter dem Fremden den Gott hören, weist er so auch auf seine göttliche Natur, welche das Hauptziel seiner thebanischen Erscheinung ist, indirekt hin.

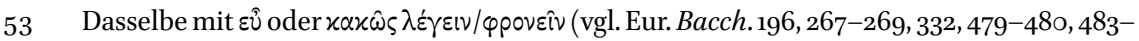
484, 1123).

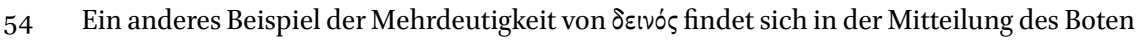
über die Taten der Thebanerinnen ausgedrückt (Eur. Bacch. 667, 716).

55 Rijksbaron (1991: 73) spricht von „a past state of affairs with present relevance“. Diese Verbform kann als Beispiel einer zyklischen Zeitvorstellung verstanden werden.
} 
für den Fremden nicht relevant. Ein anderes Beispiel: Der Gott kann anwesend

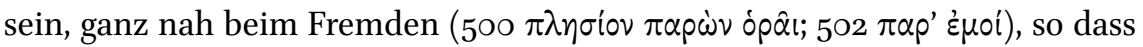

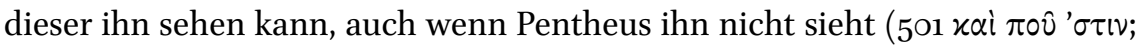

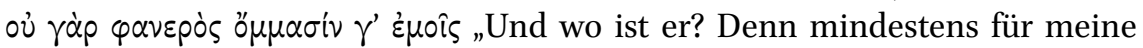
Augen ist er nicht sichtbar."). Die Mehrdeutigkeit ist hier stark mit der doppelten Natur des Fremden, der gleichzeitig auch der Gott ist, verbunden. Aber nicht nur: Auch wenn der Fremde als reiner Mensch betrachtet wird, ist die Mehrdeutigkeit verständlich. Dionysos' Anwesenheit muss nicht faktisch, tatsächlich stattfinden: Er ist da und nicht da, einfach nicht wirklich oder klar sichtbar. ${ }^{56}$

Pentheus bekommt jedes Mal die Möglichkeit, seine Ansicht und Einstellung zu ändern. Zum Beispiel erklärt ihm der Fremde nach seinen zwei ersten kritischen Bemerkungen, inwiefern seine Worte nicht „gefälscht“ oder „abgelenkt" sind. ${ }^{57}$ Diese Erklärungen enthalten zwar keine bestimmten Partikeln oder Marker. Wenn Pentheus auf die Worte hören würde, würde er aber merken, inwiefern sie kohärent sind und welche neue Information sie liefern. Vergeblich: Wie schon dargestellt, lehnt Pentheus in dieser Situation jede Art von responsiven Gesprächsschritten ab. Er hört seinen Gesprächspartner nicht, ignoriert ihn, stellt sofort eine neue Frage mit einem neuen Thema.

Dasselbe ganz am Ende der ersten Dialogstelle: Pentheus versteht nicht oder tut, als ob er nicht verstanden hätte -, dass der Fremde keine Frage mit einem Interrogativpronomen, sondern eine bloße Feststellung über Pentheus

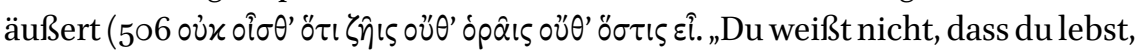
noch was du siehst noch wer du bist.") ${ }^{58}$. Sobald Pentheus ö $\sigma \tau \iota \varsigma$ हî hört, nutzt er die Chance, von sich selbst sprechen zu können, und antwortet ganz klar und präzis (im Unterschied zu dem Fremden in Antwort auf die Frage ö $\sigma \tau \iota \varsigma \varepsilon \hat{\imath}$ $\gamma^{\varepsilon ́ v 0 \zeta ~ " w e l c h e n ~ S t a m m s ~ b i s t ~ d u “ ~ i n ~ V e r s ~ 46 o): ~ E r ~ i s t ~ P e n t h e u s, ~ d a s ~ K i n d ~ A g a u e s, ~}$

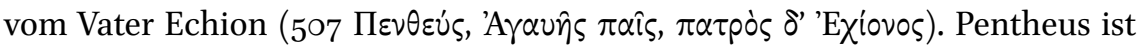
stolz, seine erhabene Mutter Agaue und seinen Vater Echion nennen zu können: Die erste verbindet ihn über den berühmten Kadmos mit der königlichen Macht; der zweite verleiht ihm als einem sogenannten Spartos, der bewaffnet aus der Erde erschienen ist, nachdem Kadmos den Drachen, der über das thebanische Land herrschte, getötet und seine Zähne ausgesät hatte, die Stärke der Erde, die kriegerische Kraft und die thebanische Legitimität. Was Pentheus

$5^{6}$ Dieselbe Interpretation gilt für die zwei Sonnen und die zwei Theben, die Pentheus nach seiner Verkleidung sieht (Eur. Bacch. 918-919).

57 Eur. Bacch. 476, 480.

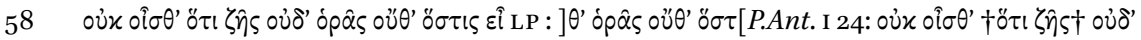

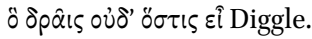


aber nicht hört, ist die mögliche Bedeutung seines eigenen Namens. Denn Пвv-

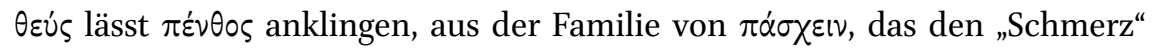
und besonders die „Trauer“ ausdrückt. ${ }^{59}$ Wie der Fremde es im darauffolgenden Vers erklärt, ist Pentheus durch seinen Namen prädestiniert, ein schlechtes

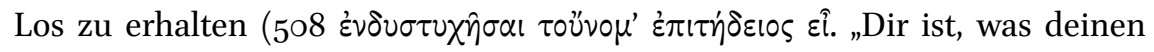
Namen betrifft, vorbestimmt, unglücklich zu sein."). Das $\delta v \sigma \tau u \chi \varepsilon \hat{v}$, das Pentheus zum Unglück und zu einem furchtbaren Tod führen wird, könnte sich

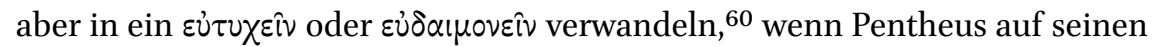
Gesprächspartner hören, seinen Sprachgebrauch akzeptieren und Dionysos' göttliche Macht anerkennen würde. Darauf reagiert Pentheus natürlich nicht. Im Gegenteil: In Vers 509 bekräftigt er seine königliche Position durch zwei deutliche Imperativformen ( $\chi \omega \dot{\rho} \rho \varepsilon ı$ und $\varkappa \alpha \theta \varepsilon i p \xi \alpha \tau^{\prime}$ ) und beendet das Gespräch.

Die Grenzen des klaren Dialogs

Die subtilen Unterschiede der Sprache versteht Pentheus also nicht. Während aller vier Dialogstellen bleibt er in seinem Sprachgebrauch der Gleiche in seinem Streben nach Klarheit und Deutlichkeit. Auch nachdem Pentheus den Vorschlag des Fremden angenommen hat, sich zu verkleiden, um in die Berge zu gehen, ändert er sich nicht. Und dies obwohl Dionysos - der Fremde ruft explizit den Gott darum an ${ }^{61}$ - eine „leichte Raserei“ über Pentheus kom-

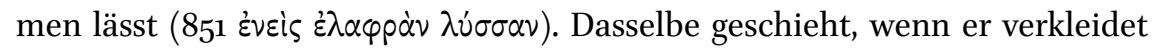
aus dem Palast tritt: Ab diesem Moment der Handlung sieht er, was er sehen

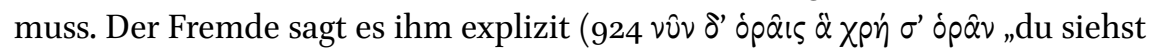
nun, was du sehen musst"). Durch seine Verkleidung hat Pentheus ein neues Sehvermögen erhalten. Es ändert aber nichts, denn er weiß dieses nicht zu gebrauchen: es ist zu wenig eindeutig. Für Pentheus zählt seine eigene Sicht, sein eigenes Aussehen. ${ }^{62}$ Und dies bis im kleinsten Detail wie eine Falte seines peplos. ${ }^{63}$

Wichtig ist ihm auch sein erster Gedanke über die Unruhe in Theben: Er bleibt bis zum Schluss überzeugt, dass die Frauen sich schändlichen Handlun-

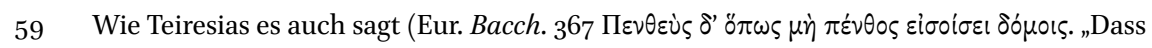
Pentheus aber keinen Schmerz in dein Haus hineinlasse!"). Eine Anspielung darauf findet

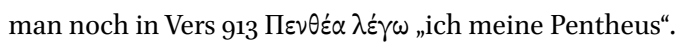

6o Zum Beispiel Eur. Bacch. 73-74.

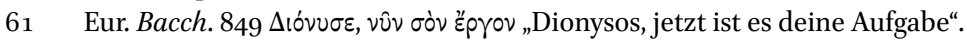

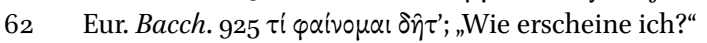

63 Eur. Bacch. $937-938$. 
gen hingeben. ${ }^{64}$ Seine Überzeugung ist so stark, dass er die Thebanerinnen nie sieht, auch wenn er vor ihnen steht. ${ }^{65}$

Pentheus versteht so wenig von der Sprachmehrdeutigkeit, die der Fremde beherrscht, er ist so selbstsicher, dass er am Ende der vierten Dialogstelle, in den letzten Worten, die er auf der Bühne ausspricht, die Sätze des Fremden unterbricht und sie auf seine Weise, nach seinem Sprachgebrauch und seiner Weltanschauung, vervollständigt. Er merkt aber nicht, dass er so seinen eigenen Tod inszeniert. ${ }^{66}$

Mit Pentheus' Tod ist das Problem, das sich in Theben abspielt, gelöst aber nicht dank einem klaren Dialog. Denn mit Klarheit und Deutlichkeit ist es unmöglich, einen solchen Gott wie Dionysos - der anwesend und abwesend, sichtbar und unsichtbar ist, und der die klaren Kategorien verschleiert und zerstört - zu erfassen und einzuordnen. Genereller gesagt ist es unmöglich, die Mehrdeutigkeit mit klaren Worten und Strukturen zu erklären und darzustellen. So stößt der klare Dialog im Leben an seine Grenzen.

\section{Bibliographie}

Benveniste, É., (1969), Le vocabulaire des institutions indo-européennes, tome 2: pouvoir, droit, religion, Paris.

Bonifazi, A., Drummen, A., \& Kreij, M. de, (2016), Particles in Ancient Greek Discourse. Five Volumes Exploring Particle Use Across Genres, Washington, D.C. http://chs.harva rd.edu/CHS/article/display/6391 (13.06.2019).

Chantraine, P., (21999 [1968-1980]), Dictionnaire étymologique de la langue grecque. Histoire des mots, Paris.

Collard, C., (1980), ,On Stichomythia', Liverpool Classical Monthly 5, 77-85 (Nachdr.:

C. Collard, Tragedy, Euripides and Euripideans, Bristol 2007, 16-30).

Di Benedetto, V., (22010 [2004]), Euripide, Le Baccanti, Mailand.

Diggle, J., (1994), Euripidis fabulae, vol. 3, Oxford.

Dodds, E.R., ( ${ }^{2} 1960$ [1944]), Euripides, Bacchae, Oxford.

Drummen, A., (2017), Language on Stage. Particles in Ancient Greek Drama, Dissertation

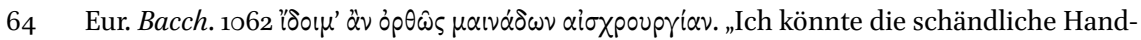
lung der Mänaden richtig sehen." Siehe auch 957-958. Im Gegenteil zu Pentheus sieht der

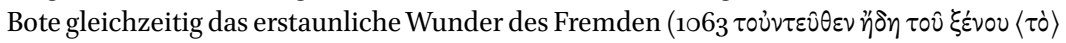
$\theta \alpha \hat{\nu} \mu^{\prime} \dot{\rho} \hat{\omega}$. „Danach sehe ich das Wunder des Fremden.").

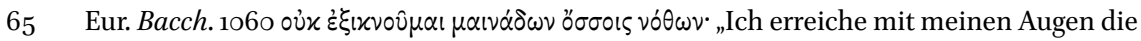

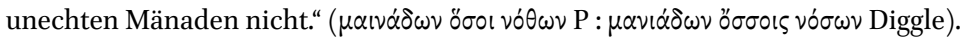

66 Eur. Bacch. 966-970. 
(Universität Heidelberg). http://www.ub.uni-heidelberg.de/archiv/23002 (14.06 $.2019)$.

Du Sablon, V., (2014), Le système conceptuel de l'ordre du monde dans la pensée grecque à

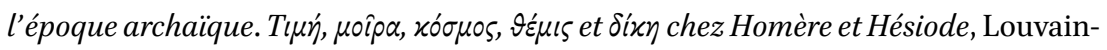
la-Neuve.

Dubel, S., (2015), ,Avant-propos: théories et pratiques du dialogue dans l'Antiquité, in S. Dubel und S. Gotteland (Hg.), Formes et genres du dialogue antique, Bordeaux, 1123.

Emde Boas, E. van, (2017a), ,Analyzing Agamemnon: Conversation Analysis and Particles in Greek Tragic Dialogue', Classical Philology 112, 411-434.

Emde Boas, E. van, (2017b), Language and Character in Euripides' Electra, Oxford.

Gross, A., (1905), Die Stichomythie in der griechischen Tragödie und Komödie. Ihre Anwendung und ihr Ursprung, Berlin.

Hall, J.M., (2002), Hellenicity: between Ethnicity and Culture, Chicago.

Hall, E., (2004), Inventing the Barbarian: Greek Self-definition through Tragedy, Oxford. Jazdzewska, K., (2014), ,From Dialogos to Dialogue: the Use of the Term from Plato to the Second Century CE', Greek, Roman and Byzantine Studies 54, 17-36.

Meibauer, J., (22001 [1999]), Pragmatik. Eine Einführung, Tübingen.

Mroczynski, R., (2014), Gesprächslinguistik. Eine Einführung, Tübingen.

Rijksbaron, A., (1991), Grammatical Observations on Euripides'Bacchae, Amsterdam.

Roux, J., (1972), Euripide, Les Bacchantes, tome 2: Commentaire, Paris.

Sacks, H., Schegloff, E.A., \& Jefferson, G., (1974), ,A Simplest Systematics for the Organization of Turn-taking for Conversation', Language $5^{\circ}$, 696-735.

Saïd, S., (1984), ,Grecs et Barbares dans les tragédies d' Euripide. La fin des différences?‘, Ktèma 9, 27-53.

Schuren, L., (2015), Shared Storytelling in Euripidean Stichomythia, Leiden/Boston.

Schwinge, E.-R., (1968), Die Verwendung der Stichomythie in den Dramen des Euripides, Heidelberg.

Seaford, R., (1996), Euripides, Bacchae, Warminster.

Seidensticker, B., (1971), ,Die Stichomythie', in W. Jens (Hg.), Die Bauformen der griechischen Tragödie, München, 183-220.

Susanetti, D., (2010), Euripide, Baccanti, Rom. 